\title{
Surface Enhanced Infrared Characterization Using Disordered Slit-Antenna Arrays for the Detection of Electrodeposited Cytochrome C
}

\author{
Gaspar Armelles ${ }^{1}$ (D $\cdot$ Alfonso Cebollada ${ }^{1} \cdot$ Daniel G. Cava $^{2} \cdot$ Julia Alvarez-Malmagro $^{2} \cdot$ Marisela Vélez $^{2}$
}

Received: 24 March 2021 / Accepted: 5 August 2021 / Published online: 28 August 2021

(c) The Author(s) 2021

\begin{abstract}
An approach which allows both electrochemical studies and surface enhanced infrared characterization of electrodeposited Cytochrome $\mathrm{C}$ is presented. This approach is based on in-plane disordered arrays of resonant slits engraved in Au substrates using focused ion beam. For light-polarized perpendicular to the slit, the reflectivity spectra of the slit arrays show dips related to the excitation of the slit plasmon resonance, whose position depends on the slit length. Due to the presence of the continuous Au layer around the slits, the very same substrates can be used to perform electrochemical studies. By varying the slit length, we have tuned the plasmon resonance to match the absorption bands of electrodeposited Cytochrome $\mathrm{C}$, demonstrating the detection of minute amounts of this protein, all the way down to a single monolayer.
\end{abstract}

Keywords Slits $\cdot$ Plasmons $\cdot$ Electrochemical $\cdot$ SEIRA

\section{Introduction}

Relating protein function with conformational changes is of great interest in biology. In the case of redox proteins, involved in many essential processes in biology, the combination of electrochemical and spectroscopical measurements can provide this valuable information [1,2]. Infrared spectroscopy is particularly interesting as it provides a nondestructive, label free, unambiguous identification of local structural protein rearrangements. However, IR cross section is low and a large amount of protein is required to provide a quantifiable and sometimes even detectable signal. Hartsein et al. [3] described that absorption of molecules on metal films can enhance the IR signal on a broad spectral range by nearly two orders of magnitude. This effect, called surface enhanced infrared absorption (SEIRA), strongly depends on the metal-film morphology. A considerable higher signal enhancement can be achieved by using micrometer long

Gaspar Armelles

Gaspar.armelles@csic.es

1 Instituto de Micro y Nanotecnología, c/Isaac Newton 8, 28760 Madrid, Tres Cantos, Spain

2 Instituto de Catálisis y Petroleoquímica, c/Marie Curie 2, 28049 Cantoblanco, Madrid, Spain plasmonic rods, often referred to as nanoantennas, under resonance conditions [4-6]. In addition to improving the enhancement, these metallic nanoantennas produce sharp plasmonic resonances which can be used to select the spectral region of interest. On the other hand, it is also well known that so-called inverse nanostructures, consisting of slits fabricated in a continuous metallic film, also feature sharp, tunable resonances over the whole mid-IR spectral range. It was recently found that the measured average SEIRA signal of slits is higher compared to their inverse rod structures for the same vibrational layer, showing their advantages for SEIRA experiments over the better-known solid nanoantennas [7, 8]. Moreover, slits present yet another advantage with respect to rods which makes them best suited for the present work: the gold surface surrounding the slits remains conducting and allows combining SEIRA with electrochemical measurements, which is of great interest to study redox proteins $[9,10]$.

In this work, we present a system based on slit-antenna arrays fabricated by focused ion beam (FIB) out of a continuous $\mathrm{Au}$ layer deposited on $\mathrm{CaF}_{2}$ substrates and with in-plane disordered arrangement. Due to the presence of the continuous Au layer around the slits, the very same substrates can be used to perform electrochemical measurements. In addition, the use of FIB as the artificial structuration technique allows us to pre-design at will the size, shape, and in-plane 
arrangement of the antennas, providing therefore with a full control of their IR response. Specifically, we carry out a slit length and concentration dependence study to analyze the enhancement SEIRA capabilities of these slit-antenna arrays. We have first calibrated this system by the use of PMMA as a reference substance, to determine the sensitivity enhancement, and then we have tested these substrates by looking at the IR spectra of Cytochrome $\mathrm{C}$ electrochemically oriented on the gold continuous film. The slits were designed to amplify the signal corresponding to the absorption of the Amide I band characteristic of proteins. This soluble redox protein of the respiratory chain has been extensively studied electrochemically [11] and spectroscopically [2] and serves as a good bench mark to test the utility of these microstructured surfaces to study the infrared signal of a monolayer of an electrochemically active protein.

\section{Methods and Materials}

\section{Slit Fabrication}

Slits were fabricated using focused ion beam (FIB) etching from a continuous $75 \mathrm{~nm}$ Au film grown on $\mathrm{CaF}_{2}(111) 1 \times 1$ $\mathrm{cm}^{2}$ epi polished substrates with a 2-nm-thick Ti adhesion layer between $\mathrm{Au}$ and substrate. The thickness of the layers was determined by $\mathrm{X}$-ray reflectometry. Au was grown by magnetron sputtering and Ti by electron beam evaporation at room temperature. A number of different patterns $200 \times 200$ $\mu \mathrm{m}^{2}$ in size are fabricated in the central part of the sample, each of them with specific slit lengths and/or concentration. All patterns have an algorithm generated disordered arrangement to minimize order-induced diffraction effects and excitation of propagating plasmons. All slits are $300 \mathrm{~nm}$ wide.

\section{Chemical Modification of the Slit-containing Au Film}

All used chemicals and materials were purchased from commercial suppliers and employed with any further purification. 4 amino thiophenol (4-ATP), tioctic acid, and horse heart cytochrome $\mathrm{C}$ (Cyt $\mathrm{C}$ ) were purchased from Sigma-Aldrich.

The $1 \times 1 \mathrm{~cm}^{2}$ gold-covered $\mathrm{CaF}_{2}$ substrate containing the slits was immersed for $10 \mathrm{~min}$ in piranha solution (3:1 $\mathrm{H}_{2} \mathrm{SO}_{4}: \mathrm{H}_{2} \mathrm{O}_{2}$ ) and then thoroughly rinsed with distilled water before exposing to ultraviolet light in a Bioforce UV/Ozone cleaner (Nanoscience) for $30 \mathrm{~min}$. Immediately after, the substrates were immersed in a $10 \mathrm{mM}$ solution (1:1 ethanol:water) of tioctic acid and 4 amino thiophenol (4-ATP) (3:2 molar ratio) and were left overnight to allow for the formation of the mixed self-assembled monolayer (SAM) on the gold surface. The modified electrodes were then rinsed with ethanol to remove physically adsorbed thiols and then with $4 \mathrm{mM}$ phosphate buffer saline (PBS) (pH 7.0) buffer. Cyt $\mathrm{C}$ binds to the mixed SAM on the gold film via electrostatic interactions. The chemically modified Au film containing the slits was used as the working electrode where electrochemical and IR measurements were performed.

\section{Cytochrome C Adsorption}

The slit-engraved $\mathrm{Au}$ modified film was incubated in $0.5 \mathrm{mg} \mathrm{mL}^{-1}$ Cyt $\mathrm{C}$ in $4 \mathrm{mM}$ PBS, $\mathrm{pH}$ 7.0. In order to adsorb $\mathrm{Cyt} \mathrm{C}$, this working electrode was cycled 20 times from -0.1 to $+0.3 \mathrm{~V}$ vs. $\mathrm{Ag} / \mathrm{AgCl}$ at a scan rate of $10 \mathrm{mV} \mathrm{s}^{-1}$. After rinsing with $10 \mathrm{mM}$ PBS, $\mathrm{pH} 7.0$, the electrochemical behavior of the immobilized Cyt $\mathrm{C}$ was characterized by cyclic voltammetry in $10 \mathrm{mM}$ PBS (pH 7.0) as previously reported Ji and Jin [12].

\section{Electrochemical Measurements}

Electrochemical measurements were carried with an Autolab PGTAT30 potentiostat controlled by GPES 4.9 software (Eco Chemie, the Netherlands). Cyclic voltammetry was performed in a three electrodes glass cell at $25^{\circ} \mathrm{C}$. The SAM modified $\mathrm{Au}$ film containing the slits was employed as the working electrode while a $\mathrm{Ag} \mid \mathrm{AgCl}(3 \mathrm{M} \mathrm{NaCl}$, from Bioanalytical System Inc.) and a flamed annealed platinum wire were used as a reference and counter electrodes, respectively.

\section{Atomic Force Microscopy Measurements}

AFM measurements were done in contact mode in air in an Agilent 5500 using a soft tip from Olympus, OMCL-RC with a spring constant of $0.05 \mathrm{~N} / \mathrm{m}$.

\section{FTIR Spectroscopy}

The reflectivity spectra were obtained using an IR microscope (Bruker Hyperion) equipped with a liquid nitrogen-cooled MCT detector, polarizers and a reflecting $15 \times$ Schwarzschild objective. The microscope was coupled to a FTIR spectrometer (Bruker Vertex 70). Absorption spectra of Cyt C, $4 \mathrm{mM}$ in $\mathrm{D}_{2} \mathrm{O}$ solution, were obtained in an IR setup from Pike Instruments using a home-made dismountable transmission cell with two $\mathrm{CaF}_{2}$ windows.

\section{Results and Discussion}

\section{Characterization of the Slit-engraved Au Film}

Figure 1 shows a sketch of the $1 \times 1 \mathrm{~cm}^{2}$ sample and AFM images of some representative patterns with different slit 
length and concentration. In order to minimize inter-slit interaction while maintaining a quality level of measured IR signal five of the patterns have a low slit concentration with the same number of slits, 1408 , in the $200 \times 200 \mu \mathrm{m}^{2}$ patterned area, which corresponds to a percentage of area covered by the slits of around 2.2\% (Fig. 1a and b). Each of these patterns had a different slit length, namely 1.7, 1.9, $2.1,2.3$, and $2.5 \mu \mathrm{m}$. In addition, two more patterns are fabricated, with a $2.1-\mu \mathrm{m}$ slit length and higher slit concentration. In particular, 2468 and 5403 slits in the patterned area, which corresponds to a percentage of area covered by the slits of $3.9 \%$ (Fig. 1c) and $8.1 \%$ (Fig. 1d), respectively.

The sample prepared shows a continuous and well adhered $72-\mathrm{nm}$-thick gold layer on top of the $\mathrm{CaF}_{2}$ surface that allows using the substrate as a working electrode to perform systematic, repetitive, and reproducible electrochemical measurements.

\section{Electrochemical Adsorption of Cytochrome C}

Cyt $\mathrm{C}$ was adsorbed to the Au surface by immersing it in a solution containing $0.5 \mathrm{mg} \mathrm{mL}^{-1}$ of the protein and subjecting the electrode to 20 electrochemical cycles from -0.1 to $+0.3 \mathrm{~V}$ vs. $\mathrm{Ag} / \mathrm{AgCl}[12]$.

Figure 2 shows the AFM images of the patterned surface after the Cytochrome was electrochemically deposited. Panel a shows that the height of the slits is $10 \mathrm{~nm}$ less than before protein deposition, indicating that a thin layer of protein might be deposited at the bottom of the slit. The AFM tip cannot access the surface of the slit walls, but the width of the slit is comparable to the one measured before protein deposition, indicating that, at most, a thin layer of protein is lining the walls and that the slit is mostly devoid of protein. The topography of the flat gold regions shown in panel $2 b$ indicates that the surface is fully and homogeneously covered by the protein. The roughness of about $3 \mathrm{~nm}$ observed corresponds well with the diameter of the monomeric protein.

Figure $2 \mathrm{c}$ shows the cyclic voltamogram obtained after the protein oriented and adsorbed to the surface. The position of the peaks observed is the one expected for the oxidation and reduction of the protein occurring by direct electron transfer from the electrode surface. Measuring the area under the oxidation peak allows estimating the amount of electrons transferred during the process, which is associated to the amount of protein adsorbed. The quantitative analysis confirmed that it corresponds to approximately one monolayer of protein being present on the surface of the electrode.

\section{Infrared Reflectivity Characterization and Sensitivity Calibration of the Slit-engraved Au Film}

In Fig. 3a, we show a sketch of the used experimental setup. An IR microscope connected to the FTIR spectrometer allows measuring reflectivity spectra in the IR range of every
Fig. 1 Sketch of the $1 \times 1 \mathrm{~cm}^{2}$ sample showing the location of the $200 \times 200 \mu \mathrm{m}^{2}$ FIBlithographed regions and AFM images of representative patterns with different slit length (1.7 (a), 2.3 (b), and $2.1 \mu \mathrm{m}$ (c), d), and slit concentration (2.2\% (a)(b), $3.9 \%(\mathbf{c})$, and $8.5 \%(\mathbf{d}))$ (b)

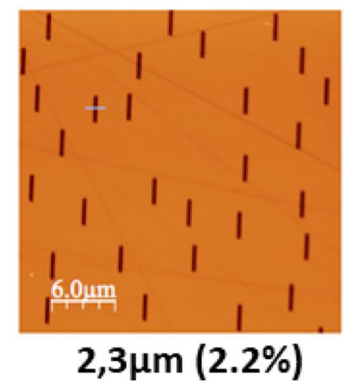

(a)

(a)

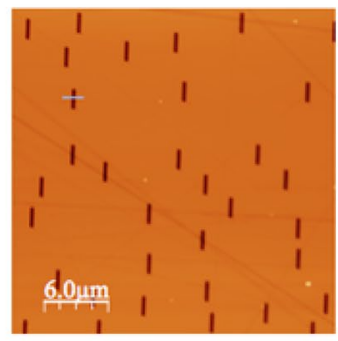

$1,7 \mu \mathrm{m}(2.2 \%)$

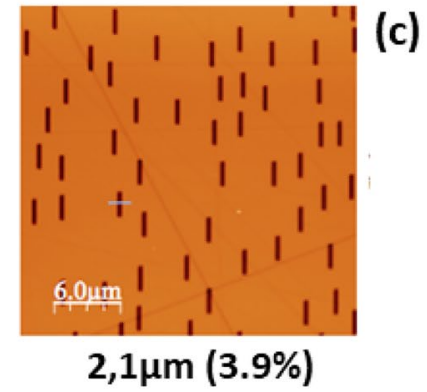

(c)

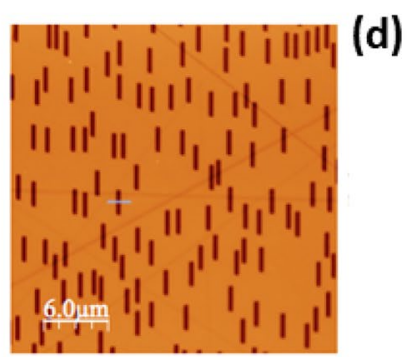

$2,1 \mu \mathrm{m}(8.5 \%)$ 
Fig. 2 AFM image of the gold surface after the Cytochrome deposition. a The height profile of one slit indicates that the protein has not filled it but is only lining its walls. b Image of a smaller region indicating that the $3-n m$ height profile is compatible with a monolayer of protein covering the surface. c Cyclic voltammogram in $4 \mathrm{mM}$ PBS, pH 7.0 showing the oxidation-reduction peaks of the Cyt $\mathrm{C}$ adsorbed to the surface. Scan rate was $10 \mathrm{mV} / \mathrm{s}$ and the temperature was $25^{\circ} \mathrm{C}$ (a)
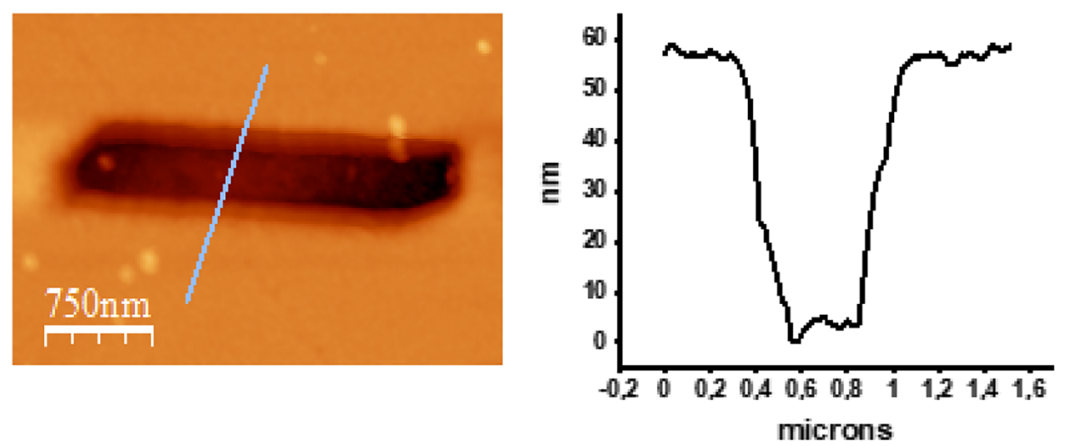

(b)
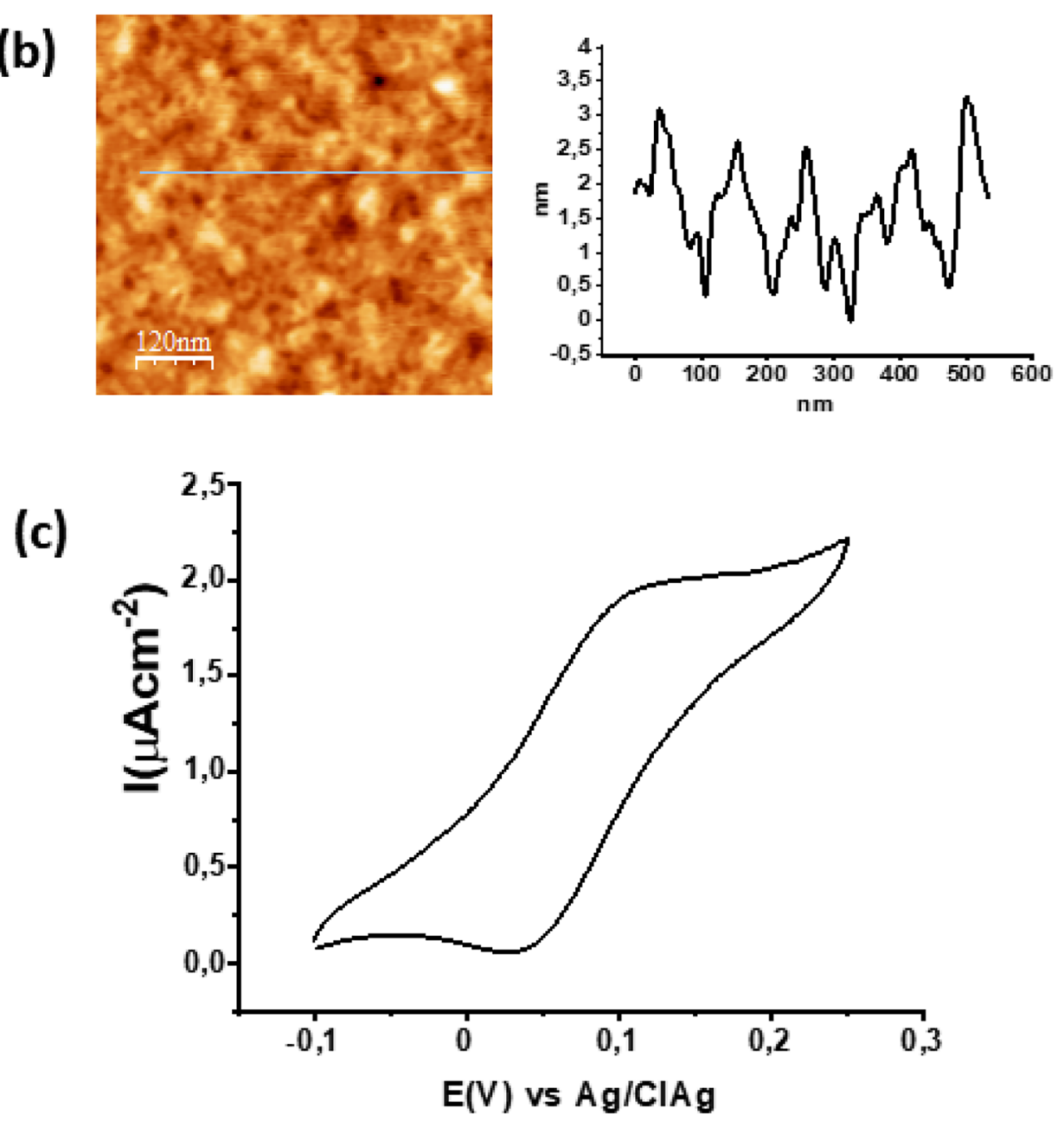

slit pattern without getting signal from the others. The slit is a plasmonic element which has resonant modes that can be excited with light-polarized either parallel or perpendicular to it, the perpendicular polarization mode appearing at longer wavelengths than the parallel one [13, 14]. As an example, in Fig. 3b, we present the reflectivity spectra for slit-engraved regions with different slit lengths, $2.2 \%$ slit concentration, and light-polarized perpendicular to the slit. As it can be seen, the spectra show a dip corresponding to the excitation of the plasmon resonance. The position of the dip depends on the slit length, shifting to lower energies as the slit length increases [15], a property exploited in the present work to tune the resonance to the spectral region of the Amide I band. On the other hand, for light-polarized parallel to the slit, no resonance is excited in any of the arrays in the considered spectral range, as it can be seen in the reflectivity spectrum of the $1.7-\mu \mathrm{m}$ long slits, represented with a dashed line.

This work is oriented to the detection of a single monolayer of protein, a challenging amount for IR spectroscopies 
(a)

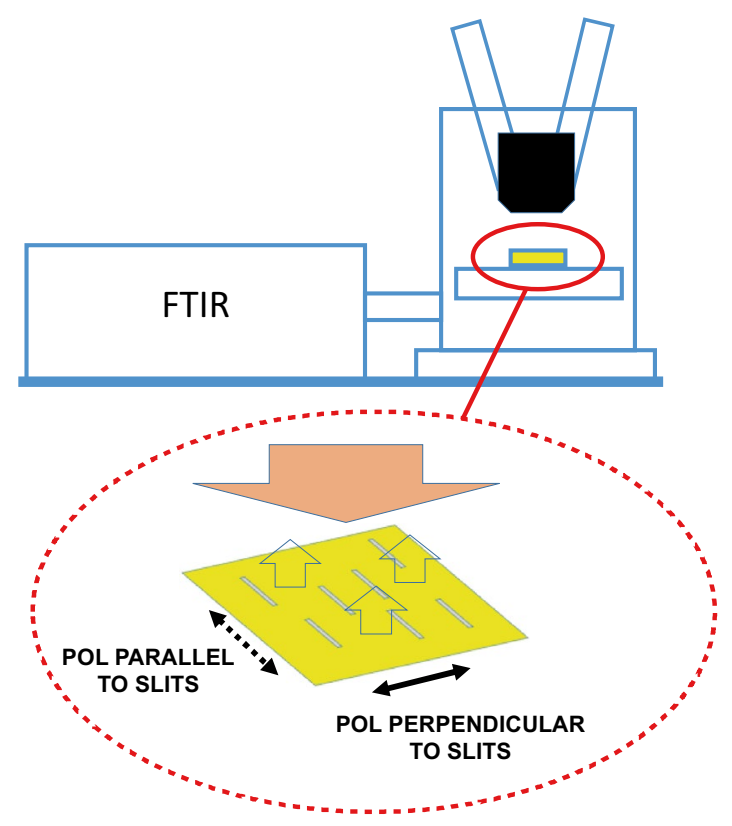

(b)

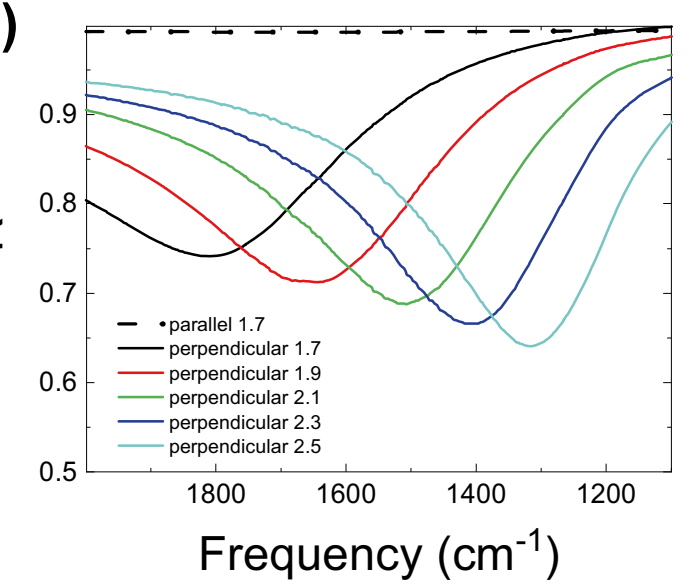

Fig. 3 a Sketch of the experimental setup. An IR microscope connected to a FTIR spectrometer allows measuring the different slits patterns. The relative orientation of the light polarization with respect to the main axis of the slits determines the excitation of the slit resonance in the considered spectral range. In this case, the slit resonance is excited by using light-polarized perpendicular to the slits. b Reflectivity spectra for light-polarized perpendicular to the slit for different slit lengths and $2.2 \%$ slit concentration. For the $1.7-\mu \mathrm{m}$ long slit, also the spectrum for light parallel to the slit is shown (dashed line)

and from which no signal was detected in the unpatterned regions of the $\mathrm{Au}$ film. Therefore, before analyzing the effect of the Cytochrome adsorption on the resonant reflectivity spectra of the slits arrays, we have characterized the SEIRA enhancement capabilities of the arrays by studying a layer of PMMA deposited by spin coating on top of the $\mathrm{Au}$ film. PMMA is a very convenient material for this purpose, since it has an intense absorption band at $1730 \mathrm{~cm}^{-1}$, which falls within the spectral range considered here. For 30-nmthick PMMA films, this absorption can be detected in the unpatterned regions of the Au film and can therefore be used to quantify the increase of the band signal due to the slit resonance. In Fig. 4, we present the slit length dependence of the reflectivity for the light polarization perpendicular to the long slit axis of the patterns with $2.2 \%$ slit concentration after PMMA deposition. The effect of depositing the PMMA layers is twofold, on the one hand there is a red shift of the slit resonance due to the change in the permittivity of the region surrounding the slit and, on the other, additional features appear at the position of the PMMA bands. As observed, the intensity and spectral shape of these features depend on the slit length.

To highlight these features, the difference between the experimental reflectivity spectra and a baseline spectra obtained smoothing the experimental ones $\left(R_{\mathrm{BL}}\right)[16,17]$, is presented in Fig. 5 in the spectral region around the $1730 \mathrm{~cm}^{-1}$ absorption band. In the same figure, we also present the spectrum of the difference (multiplied by 10) measured in the unpatterned regions of the Au film. This spectrum shows a dip at the position of the PMMA absorption band. On the other hand, the spectra of the slits regions are more complex, showing the specific properties related to the resonant SEIRA substrates. In particular, the PMMA-related feature has a spectral shape and intensity (difference between the maximum and minimum of the signal) which depends on the slit length. This

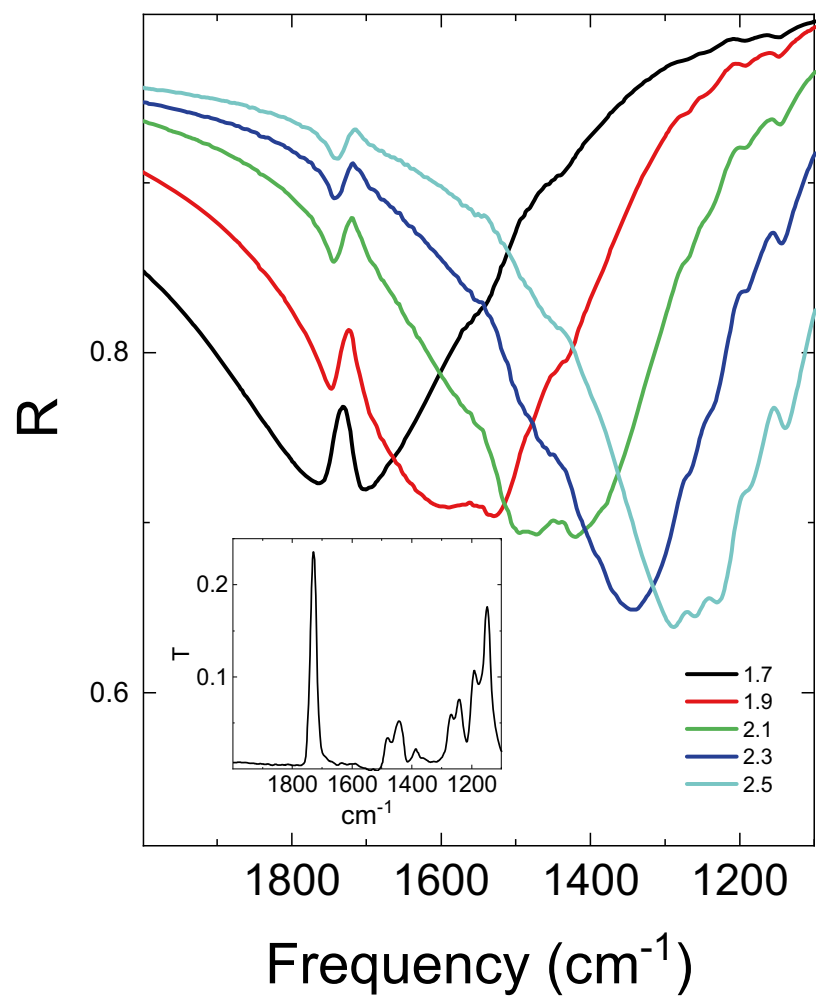

Fig. 4 Reflectivity spectra for light-polarized perpendicular to the slit long axis for different slit lengths and 2.2\% slit concentration after PMMA deposition. Inset: transmission spectrum of a PMMA layer 


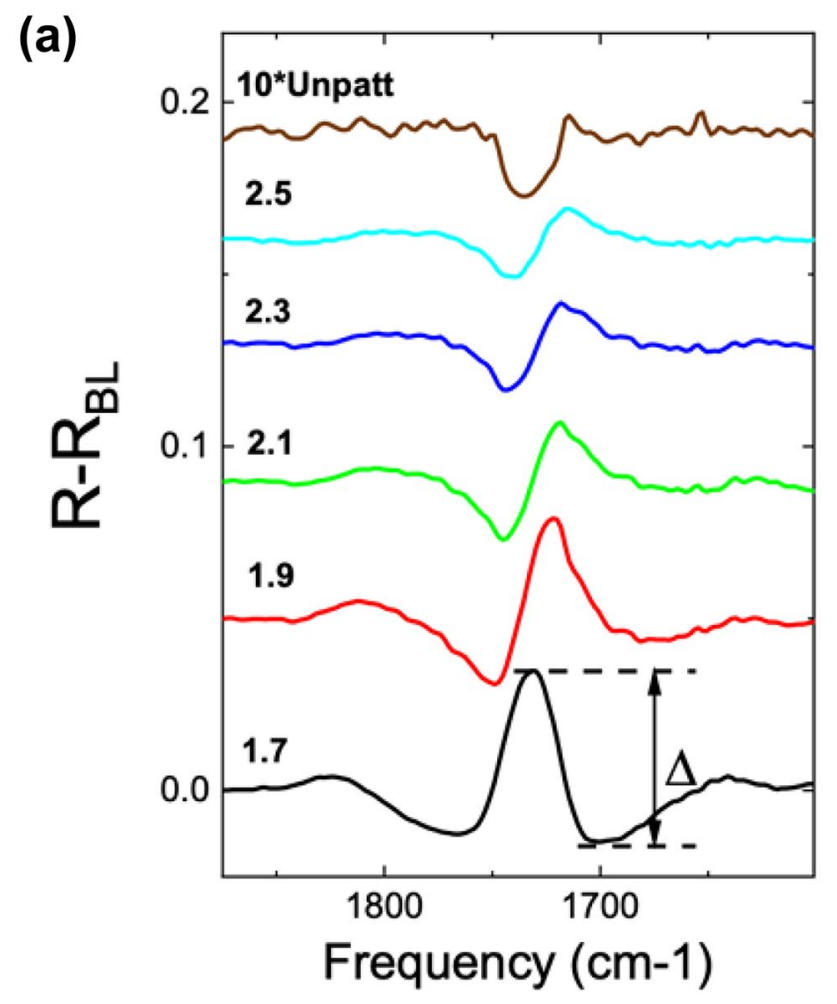

(b)

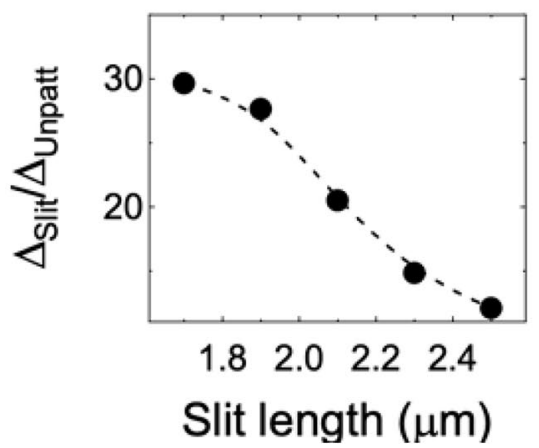

Fig. 5 a Spectra of the reflectivity after baseline subtraction. For clarity, the spectra have been displaced along the $y$-axis. The arrow represents the intensity of the PMMA-related feature. b Intensity of the $1730 \mathrm{~cm}^{-1}$ feature normalized to the intensity of the unpatterned region of the Au film as a function of the slit length. The dotted line is a guide to the eye

can be clearly seen in Fig. 5b, where we depict this intensity, normalized to the intensity of the unpatterned region of the Au film, as a function of slit length. This ratio represents the enhancement of the signal and its slit length dependence is related to the evolution of the electromagnetic field intensity in the region around the slit at the frequency of the $1730 \mathrm{~cm}^{-1}$ absorption band. This field is maximum when the slit resonance is tuned to the frequency of the band and decreases as the frequency of the resonance is detuned from it. As it can be seen, the signal enhancement reaches values of up to 30 for $1.7-\mu \mathrm{m}$ long slits. This makes this system therefore promising for the intended detection of the single monolayer of protein.
The results discussed in the preceding paragraphs correspond to patterns with a very low slit concentration. To determine to what extent the increase in slit density affects the enhancement, in Fig. 6 we present the spectra of the reflectivity with the light-polarized perpendicular to the slit after baseline subtraction for different slit concentrations and $2.1-\mu \mathrm{m}$ slit length. They were obtained from the spectra shown on the upper-left inset. The enhancements are presented in the upper-right inset of Fig. 6 as a function of slit concentration, reaching factors up to 50 for the largest concentration. As it can be seen, the enhancement increases with slit density, but it does not follow a linear trend as the slit density does (shown by the dashed red line of the figure),

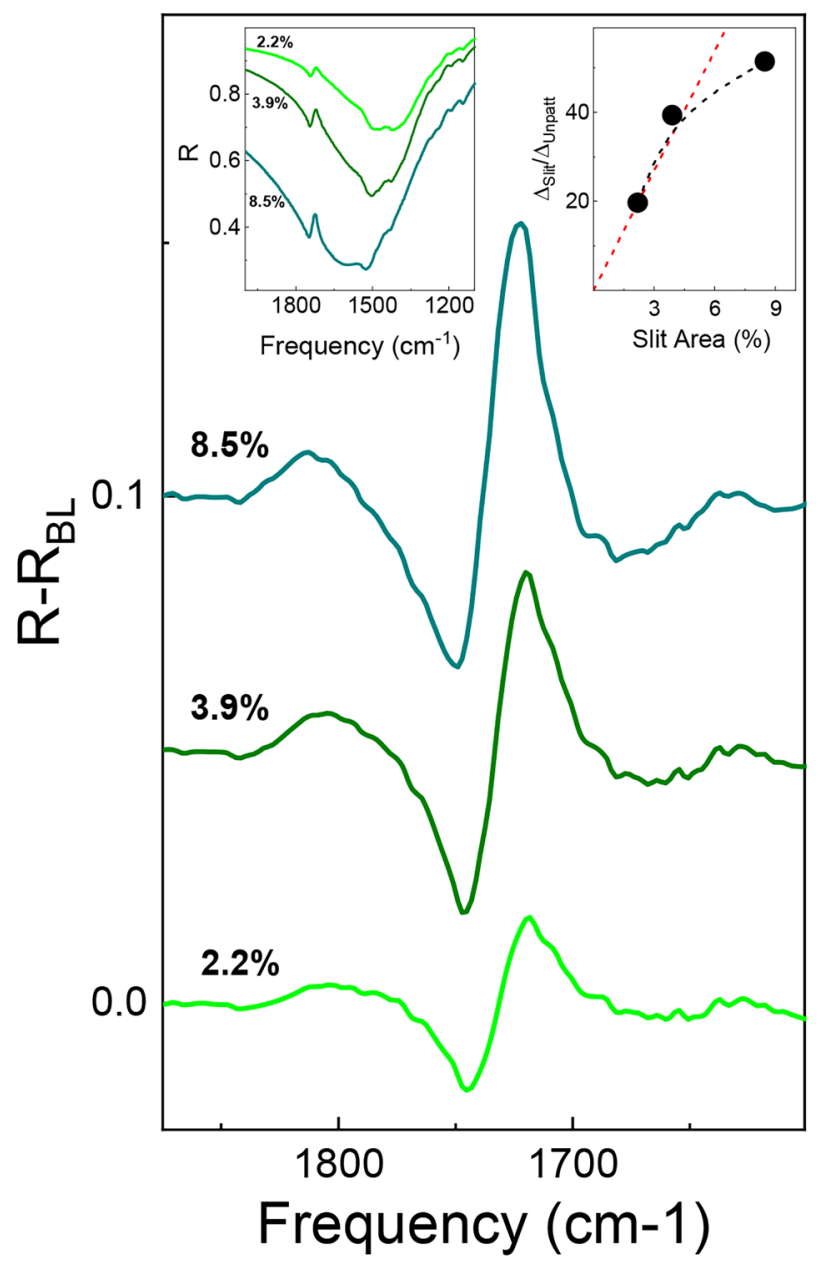

Fig. 6 Spectra of the reflectivity after baseline subtraction of the structures shown on the upper-left inset. For clarity the spectra have been displaced along the y-axis. Upper-left inset: reflectivity spectra for light-polarized perpendicular to the slit long axis $(2.1-\mu \mathrm{m}$ slit length) for different slits concentrations: $2.2 \%, 3.9 \%$ and $8.5 \%$ after PMMA deposition. Upper-right inset: Intensity of the $1730 \mathrm{~cm}^{-1}$ feature normalized to the intensity of the unpaternned region of the $\mathrm{Au}$ film as a function of the slit concentration. The dashed black line is a guide to the eye, whereas the dashed red line represents the expected increase of the enhancement assuming a linear dependence with the slit density 
rather staying below this straight line. This indicates that, even though the enhancement increases with the number of slits, as one would intuitively think, since the slits act as electromagnetic field enhancers, it occurs in a gradually slower fashion as the slit concentration increases. This is probably due to inter-slit interaction effects that modify the electromagnetic field distribution from that of an ensemble of non-interacting slits, reducing the intensity of the field. An additional indication of this inter-slit interaction effect can be also inferred from the reflectivity spectra. In this case, the position of the resonance changes with the slit concentration, and the intensity of the resonance does not follow a linear trend as the slit density does, but rather lies below this straight line, in a similar way as does the enhancement. These two effects are a clear indication of the increase of inter-slit interactions with slit concentration, which as far as SEIRA enhancement is concerned, results in a sublinear dependence of the enhancement with the slit concentration.

\section{Infrared Reflectivity Characterization of the Slit-engraved Au Film with a Layer Cytochrome C Adsorbed}

As we have already mentioned, the electrochemical adsorption of a monolayer of Cyt $\mathrm{C}$ on top of the Au surface does not produce any characteristic structure on the reflectivity spectrum of the unpatterned regions of the Au film. Similarly, when the light is polarized along the slit, and due to the absence of any resonance excitation, the reflectivity spectra of all the slits arrays do not show additional structures either. On the other hand, when the slit resonance is excited (light-polarized perpendicular to the slits), the spectra show some structure characteristic of the deposited protein layer. This structure is clearly seen in Fig. 7a, where we present the spectra after base line substation (SEIRA signal) in the region around Amide I band $\left(1700-1600 \mathrm{~cm}^{-1}\right)$. As observed, the shape of the SEIRA signal is different from that of the absorbance spectrum of Cyt $\mathrm{C}$ shown on top of the figure, which consists on a main characteristic peak at $1656 \mathrm{~cm}^{-1}$. On the other hand, the SEIRA signal has the characteristic shape of the features observed in resonant SEIRA substrates and its intensity depends on the slit length. This can be clearly seen in Fig. 7b, where we depict the SEIRA intensity as a function of slit length. The maximum of the intensity occurs for a slit length which has its resonance tuned to the frequency of the band, in this case for $1.9-\mu \mathrm{m}$ long slits.

Besides, and similarly to the results for PMMA, the slit concentration dependence of the Cytochrome features increases with the slit density, but not in a linear fashion as can be observed in Fig. 8 where we present the signal for the two extreme cases.
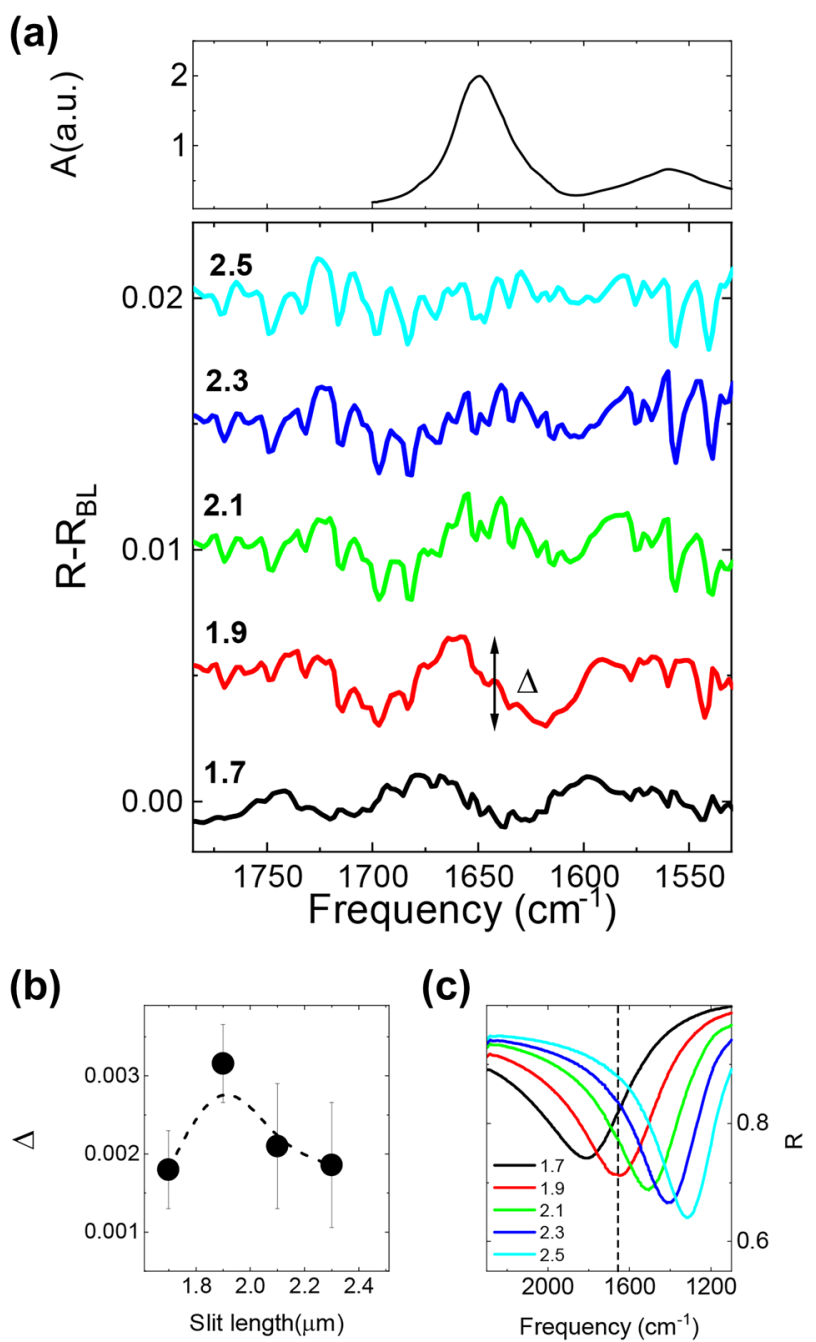

Fig. 7 a Spectra of the reflectivity after baseline subtraction. For clarity, the spectra have been displaced along the $y$-axis. The arrow represents the intensity of the feature. The curve on top of the figure is the absorbance spectrum of $\mathrm{Cyt} C$ in $\mathrm{D}_{2} \mathrm{O}$ solution. $\mathbf{b}$ Intensity of the Amide signal as a function of the slit length. The dashed line is a guide to the eye. $\mathbf{c}$ Reflectivity spectra of the $2.2 \%$ slit concentration arrays. The dashed line indicates the position of the Amide band. As it can be observed, for the 1.9- $\mu \mathrm{m}$ slit length array the slit resonance is tuned to the frequency of the band

Altogether, these results demonstrate the detection of minute amounts of electrochemically deposited Cyt $\mathrm{C}$, all the way down to a single monolayer, thanks to the use of disordered arrays of resonant slit antennas in a gold film, amounts undetectable in the absence of such antennas. These SEIRA substrates, previously calibrated using PMMA layers, allow spectrally tuning the position of the resonance to match the absorption peak of the substance to be detected. Slit concentration can also be optimized, with larger sensitivities obtained for larger concentrations. This parameter of course finds an upper limit when inter-slit interactions set in, strongly affecting spectral position of the features and 


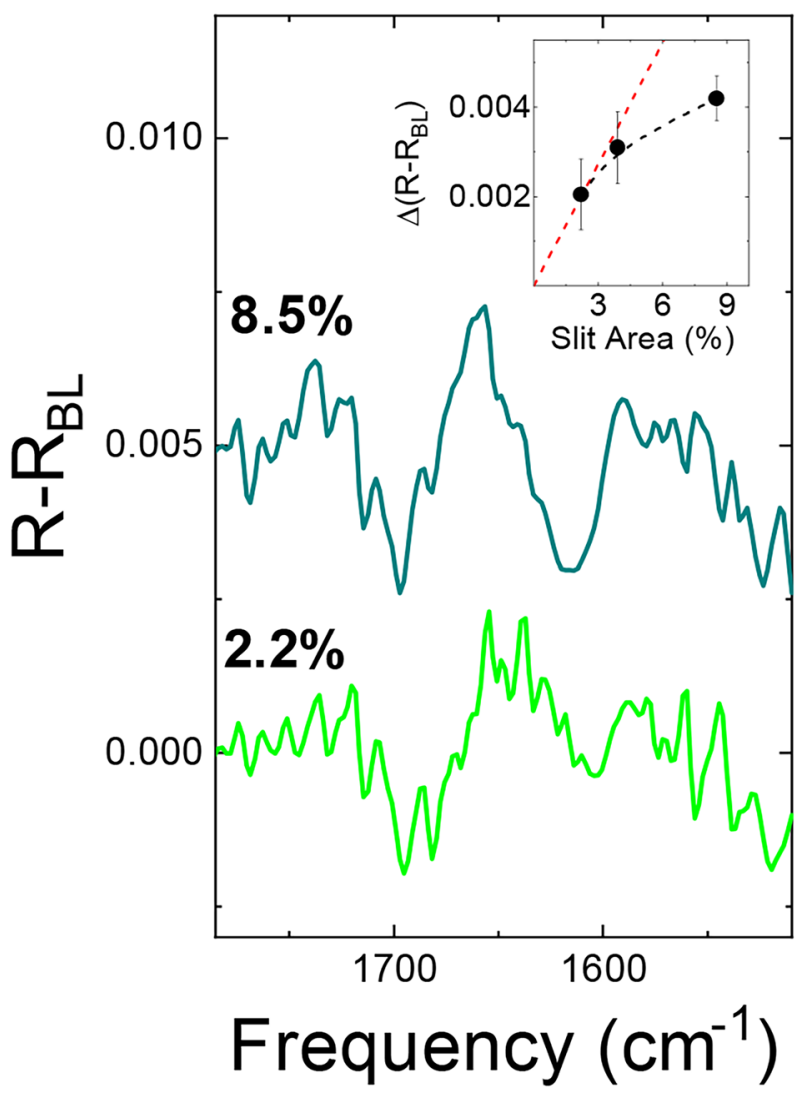

Fig. 8 Spectra of the reflectivity after baseline subtraction of the arrays region with $2.2-\mu \mathrm{m}$ slit length and $2.2 \%$, and $8.5 \%$ slit concentration, respectively. For clarity, the spectra have been displaced along the $y$-axis. Upper-right inset: intensity of the amide signal as a function of the slit concentration. The dashed black line is a guide to the eye, whereas the red dashed line represents the expected increase of the signal assuming a linear dependence with the slit density

the electromagnetic field enhancement at resonance, with a gradual reduction of the expected sensitivity enhancement due to these interactions.

The disordered arrangement simplifies the interpretation of the results as only plasmonic resonances localized in the antennas are excited. In addition, the continuous nature of the Au film surrounding the slits make it possible electrochemical experiments, required in the deposition of the selected Cyt C protein.

\section{Summary and Conclusion}

In summary, an analysis of the effect that random arrays of slits patterned on a 72-nm-thick Au layer have on the reflectivity signal of the Amide I band of a monolayer of Cytochrome C electrodeposited on the Au layer is presented. Due to the plasmon slit resonance, the single monolayer Cytochrome-related signal becomes detectable and its intensity depends on the slit length and slit concentration. This proposed SEIRA substrate can be regarded as an alternative to both ordered arrays of slits, which produce strong enhancements of the electromagnetic field but in a very narrow spectral range, and to typical SEIRA substrates based in a granular deposits of Au, with very little or none reproducibility of the detailed morphologies of the random granules. In our proposed substrates, the Au film between the slits still offers enough continuous coverage so that electrochemical experiments can be carried out. Besides, this substrate may also allow to perform in situ electrochemical studies in small areas by measuring the reflectivity from the bottom side.

Funding Open Access funding provided thanks to the CRUE-CSIC agreement with Springer Nature. We acknowledge financial support from MINECO through projects AMES (MAT 2014-58860P), Quantum Spin Plasmonics (FIS2015-72035-EXP), MIRRAS (MAT2017-84009-R), and Comunidad de Madrid through project SINOXPHOS-CM (S2018/BAA-4403). We acknowledge the service from the MiNa Laboratory at IMN and funding from MINECO under project CSIC13-4E-1794 and from CM under project S2013/ ICE-2822 (Space-Tec), both with support from EU (FEDER, FSE).

Data Availability The data/materials that support the findings of this study are available from the corresponding author upon reasonable request.

\section{Declarations}

Ethics Approval Not applicable.

Consent to Participate Not applicable.

Consent for Publication Not applicable.

Conflict of interest The authors declare no competing interests.

Open Access This article is licensed under a Creative Commons Attribution 4.0 International License, which permits use, sharing, adaptation, distribution and reproduction in any medium or format, as long as you give appropriate credit to the original author(s) and the source, provide a link to the Creative Commons licence, and indicate if changes were made. The images or other third party material in this article are included in the article's Creative Commons licence, unless indicated otherwise in a credit line to the material. If material is not included in the article's Creative Commons licence and your intended use is not permitted by statutory regulation or exceeds the permitted use, you will need to obtain permission directly from the copyright holder. To view a copy of this licence, visit http://creativecommons.org/licenses/by/4.0/.

\section{References}

1. Melin FP, Hellwig P (2013) Recent advances in the electrochemistry and spectroelectrochemistry of membrane proteins. Biol Chem 5:593-609. https://doi.org/10.1515/hsz-2012-0344

2. Melin F, Hellwig P (2020) Redox properties of the membrane proteins from the respiratory chain. Chem Rev 120:10244-10297. https://doi.org/10.1021/acs.chemrev.0c00249 
3. Hartstein A, Kirtley JR, Tsang JC (1980) Enhancement of the infrared absorption from molecular monolayers with thin metal overlayers. Phys Rev Lett 45:201-204. https://doi.org/10.1103/ PhysRevLett.45.201

4. Neubrech F, Huck C, Weber K, Pucci A, Giessen H (2017) Surface-enhanced infrared spectroscopy using resonant nanoantennas. Chem Rev 117:5110-5145. https://doi.org/10.1021/acs. chemrev.6b00743

5. Adato R, Aksu S, Altug H (2015) Engineering mid-infrared nanoantennas for surface enhanced infrared absorption spectroscopy. Mater Today 18:436-446. https://doi.org/10.1016/j.mattod.2015.03.001

6. Mayerhöfer TG, Popp J (2018) Peridodic array-based sustrates for surface enhanced infrared spectroscopy. Nanophotonics 7:39-79. https://doi.org/10.1515/nanoph-2017-0005

7. Huck C, Vogt J, Sendner M, Hengstler D, Neubrech F, Pucci A (2015) Plasmonic enhancement of infrared vibrational signals: nanoslits versus nanorods. ACS Photonics 2:1489-1497. https:// doi.org/10.1021/acsphotonics.5b00390

8. Semenyshyn R et al (2019) Resonant plasmonic nanoslits enable in vitro observation of single-monolayer collagen-peptide dynamics. ACS Sensors 4:1966-1972. https://doi.org/10.1021/acssensors.9b00377

9. Gutiérrez-Sanz O et al (2018) Catalytic activity and proton translocation of reconstituted respiratory complex i monitored by surface-enhanced infrared absorption spectroscopy. Langmuir 34:5703-5711. https://doi.org/10.1021/acs.langmuir.7b04057

10. Steininger C, Reiner-Rozman C, Schwaighofer A, Knoll W, Naumann RL (2016) Kinetics of cytochrome c oxidase from $R$. sphaeroides initiated by direct electron transfer followed by $\operatorname{tr}-$ SEIRAS. Bioelectrochemistry 112:1-8. https://doi.org/10.1016/j. bioelechem.2016.06.005
11. Jin B, Wang G, Millo D, Hildebrandt P, Xia X (2012) Electricfield control of the $\mathrm{pH}$-dependent redox process of cytochrome c immobilized on a gold electrode. J Phys Chem C 116:1303813044. https://doi.org/10.1021/jp303740e

12. Ji X, Jin B (2006) Voltammetry of immobilized cytochrome c on novel binary self-assembled monolayers of thioctic acid and thioctic amide modified gold electrodes. J Electroan Chem 590:173180. https://doi.org/10.1016/j.jelechem.2006.03.011

13. Sepúlveda B, Alaverdyan Y, Alegret J, Käll M, P. Johansson P (2008) Shape effects in the localized surface plasmon resonance of single nanoholes in thin metal films. Opt Expr 16:5609-5616. https://doi.org/10.1364/OE.16.005609

14. Yang HU, Olmon RL, Deryckx KS, Xu XG, Bechtel HA, Xu Y, Lail BA, Raschke MB (2014) Accessing the optical magnetic near-field through Babinet's principle. ACS Phot 1:894-899. https://doi.org/10.1021/ph5001988

15. Huck C, Vogt J, Sendner M, Hengstler D, Neubrech F, Pucci A (2015) Plasmonic enhancement of infrared vibrational signals: nanoslits versus nanorods. ACS Phot 2:1489-1497. https://doi. org/10.1021/acsphotonics.5b00390

16. Abb M, Wang Y, Papasimakis N, de Grott CH, Muskens O (2014) Surface-enhanced infrared spectroscopy using metal oxide plasmonic antenna arrays. Nano lett 14:346-352. https://doi.org/10. $1021 / \mathrm{nl} 404115 \mathrm{~g}$

17. Braun A, Maier SA (2016) Versatile direct laser writing lithography technique for surface enhanced infrared spectroscopy sensors. ACS sens. 1:1155-1162. https://doi.org/10.1021/acssensors.6b00469

Publisher's Note Springer Nature remains neutral with regard to jurisdictional claims in published maps and institutional affiliations. 